\title{
¿Es posible la enseñanza virtual?
}

\author{
Is e-learning possible?
}

\section{María Laura Picón*}

\section{RESUMEN}

Más de 300 millones de estudiantes alrededor de todo el mundo vieron su educación interrumpida por la expansión del covid-19. Escuelas y universidades, como nunca en generaciones pasadas, tienen las herramientas para garantizar la continuación de la educación, aún cuando sus escuelas están cerradas. Docentes, estudiantes y familias están involucradas en colaborar para que la experiencia del aprendizaje online sea exitosa. Pero ¿cuán preparados estaban los estudiantes y docentes, para sumergirse en la educación a distancia? ¿Son las clases online verdaderas clases, o se trata de una herramienta moderna de enseñanza? La educación remota, ¿reemplaza a las tradicionales clases presenciales? ¿Cómo se generan espacios de encuentro desde la virtualidad? En este artículo intentaremos mostrar cómo este es un nuevo paradigma de enseñanza que llegó para quedarse y que no se trata de un reemplazo de la presencialidad.

\section{SUMMARY}

Over 300 million students around the world saw their education interrupted due to COVID-19. Like never before, schools and universities have the tools to ensure the continuation of education, even when schools are closed. Teachers, students, and families collaborate to make online learning success; however, how prepared were students and teachers for distance learning? Are online classes really classes, or just a modern teaching tool? Does remote education replace classroom learning? How are meeting points created from the virtual? In this article, we
Palabras clave:

clases virtuales, educación a distancia, pandemia, enseñanza remota.

Key words: virtual classes, distance learning, pandemic, remote education.

Profesora de Filosofía. Doctora en Filosofía por la Pontificia Universidad Católica Argentina Santa María de los Buenos Aires. Docente Titular de Filosofía y Antropología, Lógica y Epistemología y Ética y sus fundamentos. Contacto: marialaurapicon@uca.edu.ar Orcid: https://orcid.org/0000-0002-6482-6317 
¿Es posible la enseñanza virtual? | Picón

will attempt to explain how this new teaching paradigm will endure over time and not serve as a replacement for face-to-face education. 


\section{Introducción}

Toda situación de crisis supone la caída de un paradigma² (económico, político, social...) y, al mismo tiempo, ofrece la posibilidad de construir o potenciar la aparición de un modelo alternativo. En otras palabras, somos conscientes de que ante toda crisis surge una oportunidad. En estos días, la pandemia mundial causada por un enemigo minúsculo pone en jaque nuestras seguridades básicas y las de cada persona, nos obliga a cambiar hábitos fuertemente arraigados, nos recuerda que somos vulnerables, débiles, y que no somos señores de la naturaleza, ni de la vida ni de la muerte.

Esta pandemia nos ofrece una oportunidad para reflexionar sobre nuestras acciones, sobre las conductas, sobre nuestros modos de relacionarnos, sobre el modo de enseñar y aprender. La pandemia nos limitó a permanecer en casa. En el lugar en que se habita, se trabaja, se ejercita, se estudia, se aprende, se enseña. Y es sobre estas últimas acciones que se ofrece una nueva oportunidad. Si algo queda claro es que no estamos en presencia del homeschooling, la escuela en casa, porque la intencionalidad del docente y del proyecto educativo no cambiaron, aunque sí lo hicieron las maneras de encontrarnos. La infinidad de señales que aporta el contacto físico en un encuentro presencial hoy no son viables y dan paso a nuevas maneras de medir, para dar continuidad al proceso de aprendizaje. Fue menester, sin dilación, diseñar otras maneras, otros espacios, algunos de ellos nuevos para la gran mayoría, excepto para quienes eran ya tutores online, o para quienes tenían experiencia en el aprendizaje remoto.

Estudiantes y docentes, docentes y docentes, estudiantes y estudiantes debieron volver a conectarse - nunca mejor usado el término- en una dimensión socioemocional y con un formato diferente. ¿Estamos ante nuevas estrategias educativas o ante un nuevo paradig-

\footnotetext{
2 No es el objetivo de este trabajo ahondar cuestiones acerca de la noción de "paradigma", ampliamente discutida y tratada en la epistemología y poseedora de matices muy variados en diferentes autores. En este sentido, cuando utilicemos la palabra "paradigma" en este artículo nos estaremos refiriendo a una cosmovisión, a un modo de ver el mundo - en este caso educativo-, a un modelo mental que filtra nuestras percepciones, organiza nuestros saberes en torno de un esquema, y los relaciona de un modo peculiar. En el caso que nos ocupa, el paradigma en cuestión indicará cómo enseñar cómo pensar, cómo aprender, cómo solucionar un problema, sirviendo así a las estrategias metodológicas y a los objetivos de la educación.
} 
ma? Esta pandemia, ¿hizo que nos adelantáramos al futuro de la educación o simplemente la virtualidad y la enseñanza remota son reemplazos de la tradicional clase presencial? ¿Es posible enseñar-aprender remotamente o solo podemos hacerlo desde los espacios físicos? Estos son algunas de las muchas preguntas que nos asaltan y que intentaremos responder en este trabajo.

\section{¿En qué consiste la educación?}

Para quienes estamos inmersos en el ámbito de la educación, hablar de ella se ha convertido en una obviedad que, en muchas ocasiones, nos conduce a no tener presente cuál es su esencia. Más allá de las opiniones que cada uno tenga acerca de la educación, es importante volver sobre su importancia y finalidad, pues solo así tiene sentido hablar de modelos, procesos, maneras, etc.

Según Jacques Maritain (1943),

el objeto de la educación, definido de modo preciso, es guiar al hombre en su desarrollo dinámico, en cuyo curso se forma como persona humana - provista de las armas del conocimiento, de la fuerza del juicio y de las virtudes morales- en tanto que, al mismo tiempo, va recibiendo la herencia espiritual de la nación y de la civilización a las que pertenece, conservándose así el patrimonio secular de las generaciones. (p. 10).

De lo expresado se desprenden varias cuestiones conectadas entre sí, a saber:

a. que la educación es un proceso dinámico. No es estático, fijo o asfixiado sobre una base única.

b. que forma personas en un sentido abarcativo y total, en su singularidad, atendiendo a su ser único e irrepetible.

c. que no se trata solo de circunscribir a la educación al pequeño mundo de la escuela o la universidad, como si ambas fuesen una fábrica, en la que entra un sujeto por una puerta y por otra sale dicho sujeto adiestrado. Educar supone un despertar humano.

Por lo tanto, si se trata de desplegar las potencialidades de la persona humana, lo primero que debemos recordar es que, en dicho proceso, el docente es un guía, alguien que facilitará el proceso de aprendizaje y que dispondrá la práctica de la enseñanza para facilitar ese 
aprendizaje. Este punto a veces se pierde de vista y, sobre todo en estos tiempos, encontramos docentes agobiados, invirtiendo miles de horas en planificar la enseñanza, diseñando estrategias, abordajes, herramientas, olvidando que lo que realmente importa es destacar el aprendizaje como fin primordial, pues este aprendizaje formará personas de modo integral. En palabras del mismo Jacques Maritain (1943),

la educación de la escuela y de la universidad tiene, en realidad, su mundo propio, que consiste esencialmente en la dignidad y las riquezas del conocimiento y de la inteligencia, facultad primera del ser humano. Y de este mundo propio, ese conocimiento, que es la sabiduría, es el supremo fin. (p. 27).

\section{¿Enseñanza remota?}

En estos últimos meses, a raíz de la pandemia del covid-19, la necesidad de la enseñanza remota cobró relevancia. No se trata sino de un tipo de enseñanza que se vale de herramientas del mundo online puestas al servicio del proceso educativo.

El uso de máquinas en la enseñanza es de larga data. Sin ir más lejos, aprender matemática utilizando un ábaco o una calculadora.

Ahora bien, imaginemos a ese alumno usando un ábaco o calculadora, compartiendo su proceso para sacar una cuenta, cotejar resultados con otros, consultar o preguntar acerca de algún procedimiento. ¿No es esto lo que ocurría en muchos grupos de estudio? ¿No es acaso lo que se puede proponer en un aula virtual?

Si nos remontamos a la historia, Skinner, en 1954, utilizó la máquina para "la enseñanza" ${ }^{3}$. En 1965, la Universidad de Wisconsin comenzó a dictar cursos basados en la comunicación telefónica ${ }^{4}$ y, en 1968,

La "máquina de enseñanza", diseñada por B.F. Skinner, era un artefacto que hoy veríamos como primitivo, ya que utilizaba los principios de conducta para mejorar el proceso de aprendizaje de conceptos y términos escolares. Su mayor aportación fue el concepto de "enseñanza programada", que creó con esa máquina.

$4 \quad$ En la década de 1950, las teleclases (clases por teléfono) comenzaron a atraer la atención de los educadores americanos. En aquel tiempo comenzaba a usarse el sistema conocido como "teléfono ampliado". Se trataba simplemente de un teléfono enchufado a un amplificador y a sus altavoces y micrófonos asociados. Este dispositivo hizo que las teleclases para grandes grupos fueran posibles. El uso del teléfono amplificado, que permitía a los estudiantes hablar con personalidades relevantes en diversos campos de la ciencia, se extendió a muchos centros. 
Standford fundó la Red de Televisión Educativa. Luego de la creación de internet, en 1969, la universidad de Phoenix, en 1976, ofreció el primer curso online para dar paso, en los 80 , a los primeros cursos virtuales: Learning Management System. A partir de aquí, el uso de CD-Rom como medio educativo y el nacimiento del e-Learning, en 1996, propiciaron cambios en las modalidade educativas a velocidades vertiginosas. En 2002 nació el Moodle ${ }^{5}$ y, hasta 2009, 55 millones de estudiantes habían hecho cursos online.

Antes de lo virtual, la educación a distancia ya existía, por ejemplo en sitios donde las personas vivían alejadas de una institución educativa y debían escolarizar a sus niños a través de clases de radio o programas y materiales educativos audiovisuales.

El mito de que la enseñanza remota prescinde del docente o no es auténtica enseñanza, no se funda sino en una nostalgia del pasado, y en un maniqueísmo educativo que pretende dar a elegir entre presencialidad o distancia, cuando bien sabemos que podrían coexistir y que, en cualquier caso, la modalidad es un instrumento en el proceso enseñanza-aprendizaje.

En todo proceso enseñanza-aprendizaje, el rol del maestro es vital y no puede desaparecer. No importa cuál sea la herramienta que utilice para transmitir el gusto y la pasión por el saber. Claramente, eso requiere de la presencia del docente, en su rol de tutor, motivador, guía, mediador, moderador (dependiendo de la práctica utilizada). Hay una errónea concepción de que es la plataforma o la herramienta la que educa. Así como no se puede educar una máquina porque no es un sujeto educable, tampoco ésta puede "educir" del alumno su potencialidad. Al igual que en la presencialidad, el docente es un elemento indispensable en el proceso educativo, solo que, en la modalidad remota, la formulación de una propuesta pedagógica requiere de un sistema de acompañamiento distinto, en el que los materiales didácticos no son simples medios auxiliares, sino elementos fundamentales para el proceso enseñanza-aprendizaje.

En 2002, el australiano Maritn Dougiamas creó una plataforma de enseñanza virtual, hecha con software libre, y la llamó "Moodle". Hoy la usan millones de profesores en todo el mundo para automatizar tareas y comunicarse con sus alumnos. 


\section{Virtualidad vs. presencialidad}

Si hay un debate en el tapete por estos tiempos es si la escuela virtual puede educar o no. Otra formulación posible de la misma cuestión es si esta escuela reemplaza a la presencial, pregunta cuya respuesta negativa tiene miles de adeptos desesperados por volver a la presencialidad. Ante todo, debemos aclarar que las resistencias con la virtualidad no son por la virtualidad misma, sino por tratarse de una enseñanza a distancia o remota. Para ponderar las fortalezas y debilidades de cada una hay que tener presente, en primer lugar, que la virtualidad o la presencialidad son modalidades y, como tales, conllevan métodos diferentes.

La enseñanza a distancia o remota tiene dos pilares sobre los que se asienta: la sincronía y la asincronía. En la etapa sincrónica, los alumnos participan de una clase en vivo, mediante un entorno virtual que les permite compartir el espacio con el docente y otros estudiantes, respetando días y horarios específicos. La asincronía digital, por otro lado, permitirá a ese alumno abordar contenidos on demand, es decir, podrá interactuar con el contenido a su ritmo.

Solo señalando esto ya estamos en presencia de una diferencia importante respecto de la enseñanza-aprendizaje presencial: no se comparte el mismo espacio físico ni se realiza en los mismos tiempos y horarios. Ahora bien, ¿cuáles son los motivos concretos por los cuales parecería existir tanta resistencia a la virtualidad?

En primer lugar, es justo marcar que la situación de pandemia no dejó tiempo para que muchos docentes y estudiantes, de todos los niveles, pudieran estar a la altura de abordar una enseñanza remota. ¿Cuán preparados estaban los docentes para abordar una educación a distancia? ¿Todos los estudiantes tienen la posibilidad de conectarse desde sus casas? No es nimiedad que toda esta transición instantánea se realizó y realiza en un contexto emocional endeble (para el docente y el alumno), que complica los procesos de enseñanza y aprendizaje. La casa en la que se estudia o se enseña es la casa en la que se vive, se trabaja, donde también otros aprenden y ejercitan. Se puso de manifiesto diferencias en las habilidades para administrar los recursos, por parte de los alumnos y de los docentes. Otras dinámicas de aprendizaje debieron ser dejadas de lado, ya que solo tenían sentido en el ámbito de la presencialidad, para incorporar nuevas dinámicas, aún no lo su- 
ficientemente testeadas. Esta adaptación en tiempo récord claramente plantea falencias de muchos docentes, pero también de muchos alumnos. Se suele decir comúnmente que los jóvenes son nativos digitales. $\mathrm{Y}$ aquí me permito disentir con quienes afirman esto. Ser nativo supone hablar un lenguaje. Nuestros jóvenes no hablan Java, html o Pascal. Nuestros jóvenes son buenos usuarios de algunas tecnologías, las cuales - las más de las veces- no pueden configurar de modo óptimo para sacarles el provecho adecuado. Que sean asiduos usuarios de las redes sociales no los convierte en usuarios expertos de una plataforma digital de aprendizaje. Mi experiencia como docente a distancia me lo dice diariamente, por lo que no se trata solo de falencias docentes sino del encuentro de estas falencias con las de un alumno al que sorprendió una plataforma digital para estudiar.

Volviendo al punto, si bien esta mudanza repentina puso de manifiesto falencias, no todo es negativo.

Hay que entender que las reglas del mundo digital son diferentes a las de la enseñanza presencial. Por ello la insistencia en que lo virtual reemplace a lo presencial es cuasi hegeliana. Lo presencial no es virtual y lo virtual no puede ser presencial.

"No debe caerse en la simpleza que la enseñanza a distancia es tener una plataforma en internet", sostiene Kato Asato, director regional y fundador de la sede e-learning de la Escuela Superior de Creativos Publicitarios. "Implica la construcción de la arquitectura de un sistema educativo que utiliza multiplataformas digitales para alumnos con comportamientos distintos al alumno presencial", dice Rocca (2020, s/p).

\section{El desafío de la virtualidad o las clases remotas}

Las clases impartidas de modo remoto son un instrumento de enseñanza-aprendizaje en las cuales docentes y alumnos pueden interactuar, comunicar, visualizar y discutir, organizar trabajos colaborativos, en un ambiente digital en tiempo real (sincronía) o en tiempos on demand. En algunos casos, las clases pueden incluir un software para videoconferencias que permitirá que cada usuario esté conectado al mismo tiempo a través de internet. En otros, estas videoconferencias permitirán replicar lo que se podría haber hecho en 
una clase presencial, pero lo cierto es que proponen otros modos de interacción diversos, como puede ser el chat, un foro, realidad aumentada, etc.

Si posee algunas fortalezas esta modalidad, son que se estimula al docente a desarrollar actividades grupales y colaborativas, y, por otra parte, favorece la utilización de dispositivos distintos para realizar el aprendizaje de modo interactivo y atractivo.

En los últimos años hubo un blend entre clases virtuales y presenciales, desarrollando el programa del curso en forma mixta. En cualquiera de los casos, si algo ha aportado y aporta la clase virtual es que permite la formación continua y esto se puede constatar en la cantidad de meetings y congresos que han tenido y tienen lugar utilizando esta modalidad, la que permite el intercambio y la adquisición de nuevos conocimientos.

Es importante comprender que, cuando se habla de clases remotas o a distancia a través de medios digitales, lo que ha cambiado es el ámbito, pero no los dos elementos esenciales para que haya escuela, universidad o conocimiento del tipo que sea: maestro y discípulo.

Cuando en el ámbito universitario, en estos tiempos, escucho resistencias sobre la modalidad virtual, me viene a la memoria la definición de universidad que aprendí cuando ingresé a ella: comunidad de profesores y alumnos que se consagran al cultivo de la verdad mediante la investigación, la docencia y los diversos servicios que brinda.

Si analizamos esta definición, en ella no se menciona las modalidades, el lugar físico, las herramientas, sino que se señalan los elementos esenciales: maestros, discípulos y la finalidad, que es la búsqueda de la verdad, lo cual — como señalaba Maritain — no es otra cosa que educar personas de modo integral.

¿Por qué no habría de funcionar una clase virtual? ¿Por qué no sería un modo lícito para desarrollar el proceso enseñanza-aprendizaje?

La clase virtual es totalmente viable, ya que en ésta el docente no solo es capaz de transmitir su sabiduría, sino que sus características facilitan que pueda compartir información por múltiples canales, despertando en el alumno inquietudes diversas y abriéndole paso a horizontes nuevos que van más allá de una bibliografía física. 
Permite también desarrollar colaboración entre colegas y propone profundizaciones en momentos personalizados, fuera de la plataforma escolar. Se pone en marcha así un aprendizaje adaptativo, que permite al estudiante aprender a aprender, volviéndose un miembro activo en el proyecto formativo.

Por lo tanto, una clase virtual necesita, igual que una clase presencial, de los actores (maestro- discípulo) y de la claridad del fin al que se desea arribar. Quiero entender que aquellos que se resisten a la virtualidad no entendieron bien de qué se trata, y se han escudado en la idea de que enseñar-aprender mediante esta modalidad es muy difícil. Ciertamente que es una tarea a la que hay que adaptarse y que esta pandemia no ha dado el tiempo suficiente, pero, nobleza obliga, no se trata de falencias de la modalidad sino de quienes se valen de ella.

Antonio Vigilante (2020), en su artículo "Cos'è una classe virtuale (e perché deve essere libera)" dice:

$¿$ Cosa vuol dire fare didattica a distanza? Non significa, certo, scrivere sul registro elettronico le pagine da studiare e i compiti da fare. Ma non significa nemmeno mandare videolezioni o fare lezioni in videoconferenza. Massima stima per chi lo fa, ma manca qualcosa. Manca una riflessione su cosa è l'apprendimento e cosa è la scuola ${ }^{6}$.

Y en este punto es bueno detenernos. Si algo está remarcando el autor antes citado es que la virtualidad tampoco trata de nuevos recursos, apilados como en un repositorio, para que el alumno haga con ellos lo que pueda. Es menester no perder de vista que el proceso educativo tal como se mencionó al principio- tiene por finalidad desplegar las potencialidades de la persona humana de modo integral y que educar es educir y ayudar a actualizar las potencias que ésta posee. Por lo tanto, si la virtualidad recibe tantas críticas, es porque se la entiende fuera del proceso educativo, entre otras cosas, por la falta de capacitación de los docentes.

6 ¿Qué quiere decir "didáctica a distancia"? No significa, ciertamente, escribir sobre un registro electrónico las páginas a estudiar y las tareas para hacer. Pero tampoco enviar videos y hacer lecciones en video-conferencia. Mi reconocimiento a quienes lo hacen, pero falta algo. Falta una reflexión sobre qué cosa sea aprender y qué sea la escuela (traducción propia). 
Se puede entender que, luego de muchos años de realizar una tarea de un modo, sintamos que somos peces en el agua, y que tener que adaptarnos a otros ámbitos puede ser una empresa dura que nos saca de nuestras “zonas de confort”. Pero en la presencialidad también se supone que el docente se agiorna, se adapta, cambia y se renueva, y no queda anquilosado en sus recursos. Cada curso y cada grupo humano es un desafío, que requiere que adaptemos las herramientas y los recursos, aún cuando en otro grupo humano hayan tenido éxito. Pero no endilguemos a la virtualidad características que no posee. Si el fin de la educación está claro y están los actores principales, nada impide que esta modalidad no sea adecuada.

\section{Comparando la presencialidad y la virtualidad}

El refrán popular dice que las comparaciones son odiosas. Sin embargo, un interesante aporte se desprende de ellas, sobre todo cuando no hay ánimo de sea la ocasión para destruir a uno de los términos comparados.

A continuación, enumeraré algunos componentes que se presentan en ambas modalidades de clase, con el fin de clarificar aún más la cuestión.

\section{El maestro o docente}

Cuando planteamos los roles dentro de la educación, es importante hablar del maestro o el docente (podríamos también referirnos al profesor). Los tres términos hacen referencia a algo más que un simple actor. Designamos "maestro" a aquel que no solo conoce algo que transmite, sino que es capaz de transmitir el gusto por eso que sabe. El término docente viene del verbo docere, el que enseña (no instruye), el que educe (educa). Por último, el profesor es quien profesa, quien tiene una inclinación o sentimiento fuerte hacia algo, en este caso al conocimiento.

Cada vez que nos hallamos frente a un maestro, docente o profesor, estamos en presencia de algo más que un simple instructor o adiestrador. Estamos frente a alguien que, con su rol activo, no solo transmite, sino que pretende que, mediante su transmisión aquel que escucha se transforme. Es un guía, alguien que se detiene y es capaz de contemplar a su auditorio y de estimular el pensamiento crítico que le da calidad al aprendizaje. En este sentido, tanto las clases virtuales como las presenciales pueden tener como responsable a un maestro, docente o profesor 
guía, o a un simple adiestrador. ¿Cuántas experiencias hemos tenido de presuntos docentes que, sin reparar jamás en los participantes de sus clases, repetían, solos, tratados interminables, sumamente completos, pero sin alma y sin corazón? ¿Cuántos pretendidos docentes no proveen feedback a los trabajos entregados, sea la modalidad presencial como virtual, y cuántos otros estimulan y fomentan actitudes de profundización en cualquiera de ambas modalidades? La virtualidad no es sinónimo de docente pasivo. Si el docente es pasivo, entonces no está enseñando y esto también se puede dar en el espacio físico.

\section{El alumno o estudiante}

Otro de los roles que encontramos en un proceso educativo es el de alumno o estudiante. En algunos círculos docentes, el concepto "alumno" no está bien visto, porque sostienen que a-lumen significa sin luz y que la expresión correcta sería "estudiante". Sin embargo, sería interesante analizar correctamente el origen de esta palabra, que proviene del latín alumnus, la cual está emparentada al verbo alere, que significa "alimentarse". Por lo que la real acepción de la palabra haría referencia a aquel que se ha alimentado, como quien aprende para crecer, aludiendo así al sentir profundo de la educación como un proceso activo, en el cual el alumno se alimenta en la participación con el docente. Este mismo rol activo lo pone de manifiesto el término "estudiante", participio activo del verbo "estudiar", es decir el que está tomando parte del estudio.

En esta línea, un alumno es un participante activo, pues quien se alimenta puede hacerlo a partir de una comida realizada por otro y hasta premasticada por otro (pensemos los pichones de aves a los que su progenitor les facilita el alimento procesado), pero todo el acto de nutrición es propio.

La modalidad virtual o presencial requiere de un estudiante con un rol activo. Cada uno es causa eficiente del propio conocimiento, ya adquirido a través de la relación con un docente y compañeros de modo físico, ya través de actividades de la plataforma online que permitan mantener una comunicación fluida.

No se puede negar que muchas veces los participantes de la educación online tienden a sentirse abandonados, pero dicha sensación se debe a una falta de capacitación del estudiante para aprovechar las instancias de relación que la virtualidad propone, y/o a falta de capaci- 
tación del docente, que no conoce o cuenta con las herramientas para diseñar estos espacios.

También es cierto que la irrupción de la modalidad virtual, por las exigencias de los tiempos que corren, enfrentó a muchos alumnos a la insuficiencia de medios tecnológicos para abordar las clases. Pero esto no es una dificultad de la educación remota, sino de la ausencia de políticas que apunten a la igualdad de oportunidades.

¿Qué alumno no ha tenido la triste sensación de que su docente monologaba y no generaba espacios de intercambio? ¿Cuántos docentes hemos constatado que frente a nosotros estaba alguien que no quería estar allí y que, por más que hiciéramos, el conocimiento no era adquirido porque el sujeto no deseaba activar? En ambas modalidades, la participación del alumno es indispensable y, para ello, éste debe contar con las herramientas para hacerlo. En caso de tener que compensar esa falta, tanto la modalidad presencial como la virtual se ven interpeladas a realizar los ajustes correspondientes, de modo que el proceso educativo pueda alcanzar su fin.

\section{Métodos de enseñanza}

Sin ser expertos en la materia, es claro que este punto difiere en una $u$ otra modalidad. Si bien hoy los procesos de enseñanza-aprendizaje en la presencialidad ya no están limitados a los niveles de creatividad de los docentes, porque la inclusión de las perspectivas de los alumnos enriquece la clase, lo cierto es que las herramientas didácticas que ofrece la virtualidad son muchísimas. Diseñar una actividad para autoevaluar aprehensión de contenidos a través de un juego, en la presencialidad suponía horas de preparación por parte del docente. Desde la irrupción de la virtualidad, aun en las clases presenciales, la modalidad mixta permitió al docente utilizar herramientas metodológicas de modo más rápido, modificando plataformas prediseñadas y presentando contenidos, valiéndose de recursos atractivos y hasta semejantes a los utilizados por los alumnos fuera de la escuela para el esparcimiento.

La posibilidad de tocar o curiosear sobre una plataforma digital, que permite repetir el intento aun cuando estuviera errado, ayuda también emocionalmente al alumno a no temer equivocarse y a entender que el error forma parte del proceso de aprendizaje: aprende a aprender. 
Esta cuarentena habilita saberes nuevos y hace un especial hincapié en la capacidad que la formación a distancia desarrolla para el aprendizaje en general: el "aprender a aprender", algo difícil y que los educadores están intentando inculcar a los niños desde pequeños. Se trata de "sostener la atención en una tarea sin distraernos, de hacer foco, de trabajar la persistencia". Son habilidades para la vida que se van desarrollando de a poco y cuya adquisición representa toda una oportunidad. (Rocca, 2020, s/p).

\section{Material}

Desde hace algunos años, la incorporación de la digitalidad en las clases tradicionales las transformó en clases mixtas. Sobre todo, se vio esta transformación en lo que al uso de materiales respecta. De materiales extensos o limitados a la comunicación verbal, no verbal o escrita, se pasó la innovación aportada por la TIC, poniendo al alcance material proveniente de contenidos audiovisuales, foros, mensajerías internas, correos, imágenes o herramientas creativas. Ofrecen un sinnúmero de recursos que el alumno puede elegir según sus afinidades, armando así una trayectoria personal y a medida. Claramente, la virtualidad facilita la obtención del material, pues tenemos a disposición una biblioteca ilimitada, abierta los 365 días del año en todo el mundo. Obviamente, esta amplitud de recursos exige al docente hacer una buena selección, pero evita reversionar, ya que mucho de lo que requiere está hecho y es óptimo.

Esta esfera pedagógica requiere volver a situarse en el objetivo principal: proponer instancias de aprendizaje que mantengan un grado de participación alta, y con ello el protagonismo de los alumnos. La idea no es tener a los estudiantes largas horas conectados dando cátedra; así tampoco lo haríamos en una situación presencial. Es muy importante que la consigna de trabajo tenga claridad en los objetivos de aprendizaje, transparencia en las expectativas de logro para estudiantes y, sobre todo, fomentar la participación, promover conversaciones, hacer que el material del curso sea relevante para alentar las contribuciones individuales. (Pelliccia, 2020).

\section{El tiempo}

Quedó atrás el horario fijo (excepto en un encuentro de videollamada) y nos encontramos con tiempos flexibles. Esto es otro gran logro. La 
asincronía ofrece la ventaja de ajustar el horario al alumno. Se ha estudiado mucho el hecho de que hay personas que rinden más temprano, o a la noche, o estudiando dos horas y luego tomándose un tiempo para la recreación. Esto permite aprovechar también el conocimiento. No importa el motivo, porque la virtualidad permite sumarse al aula en cualquier horario para aprender.

Esto trae aparejado algunas dificultades:

a. Requiere cierta disciplina de parte del estudiante, que él deberá ir aprendiendo y que se puede ir enseñando mediante pautas o recomendaciones del docente.

b. Requiere, por parte del docente, establecer tiempos u horarios para guiar, responder preguntas o aclarar temas. El docente virtual no es un docente 24 horas x 365 días, como tampoco lo es en la modalidad presencial.

Mientras en las clases presenciales el tiempo y el espacio era el de la clase y en la clase, la educación virtual lo flexibiliza.

El reto es que los alumnos puedan aprovechar las ventajas de la virtualidad y que el docente pueda estimular su uso adecuado.

\section{El espacio}

Es casi una obviedad señalar la diferencia respecto del espacio en una y otra modalidad. Sin embargo, es interesante hacer foco en que la virtualidad no es una traducción de la presencialidad. Como se mencionó, es necesario identificar las oportunidades que cada una de las modalidades ofrece. No se puede negar que hay espacios físicos que disponen mejor o peor al proceso de enseñanza-aprendizaje.

The spaces in which we work, live and learn can have profound effects on how we feel, how we behave, how we perform ... spaces can also limit the possibilities of our activity, restricting us to old modes of working and thinking7. (Watson, 2007, p. 260).

Los espacios en los cuales trabajamos, vivimos y estudiamos pueden tener profundos efectos en como nos sentimos, como nos comportamos, como rendimos... (los) espacios pueden también limitar las posibilidades de nuestra actividad, limitándonos a antiguos modos de trabajo y pensamiento (traducción propia). 
Personalmente, me ha tocado enseñar en lugares donde todo estaba dispuesto para realizar la scholé y sitios en los que el entorno desfavorable auguraba con suerte la aprehensión de algunos pocos contenidos. En ambos casos experimenté que, si bien las condiciones interferían para mejor o peor, el éxito no era directamente proporcional.

El encuentro con la verdad y el conocimiento que supone el proceso enseñanza-aprendizaje no depende del espacio físico, aunque el espacio físico condiciona (no es lo mismo aprender en casa, donde toda una familia convive en cuarentena, con los efectos emocionales y psicológicos que esto conlleva, que hacerlo en la misma casa, con la libertad de poder cortar y dar una vuelta en bicicleta, en un ámbito emocionalmente propicio).

Enseñar y aprender es una relación y, como tal, implica la construcción de un sistema educativo en el que el encuentro sea posible. Cada modalidad supone "espacios" de encuentro distintos, con comportamientos diferentes para la socialización.

Pensar la tecnología no solo como un canal de intercambio de información permite mucho más que escuchar una explicación: es brindar la oportunidad para que se genere intercambio significativo.

\section{La relación educativa}

Muchos han visto y vivido la irrupción de la tecnología en la educación como un peligro, una amenaza para el correcto proceso educativo. La supuesta pérdida de la relación entre educador y educando, en tanto que personas, ha sido el argumento básico esgrimido por los detractores de la entrada de la tecnología en la dinámica educativa. Hace tiempo que sabemos que lo que define la relación entre las personas no es tan solo el medio que usan para hacerlo, sino, sobre todo, la capacidad para relacionarse. Y, para hacerlo, no usamos solo la presencia física, el cara a cara.

El acto educativo es una relación, sea cual fuere el medio utilizado. Si retomamos la definición de "universidad", por ejemplo, se pondrá de manifiesto que el peso está puesto en la relación, en el encuentro alumno- docente; se da en un encuentro intencional, en el contexto escolar (físico o virtual) en el que se producirán un conjunto de experiencias que orientarán el proceso enseñanza-aprendizaje. 
Un ejemplo de estos tiempos es la sesión en vivo, cuya finalidad es conectar varios participantes para reducir la brecha comunicativa entre ellos. En este tipo de prácticas se produce una relación que implica tres factores: el docente, el cognitivo - por parte del alumno- y el social. Dichos elementos, ya sea en el modo presencial o en línea, producen una experiencia educativa completa, alimentando la relación mencionada.

El peligro de concebir la relación educativa solo bajo la forma de una comunicación entre el alumno y el profesor en medio del conjunto del aula física, sin abordar también las características de los compañeros, genera una pérdida de horizontes. La relación educativa se sitúa en un sistema de relaciones más amplias que las constreñidas al espacio escolar, pues engloba las relaciones sociales generadas a partir del aula, las relaciones entre el aula, la escuela y la sociedad, y las relaciones de los sujetos con el saber y la lectura. Todas estas relaciones sociales ampliadas pueden desplegarse también en, por y desde la virtualidad.

\section{La evaluación}

Otro de los grandes miedos a la hora de proponer la virtualidad como modalidad es ¿cómo saber si el alumno aprendió o no un contenido? Esta pregunta, que se hace en el contexto de la virtualidad, es idéntica a la que realiza y realizó cualquier educador de la historia. Incluso más: supone una discusión anterior, esto es, ¿para qué es importante entender si una persona aprendió o no un contenido? Y la pregunta, en cualquier caso, nos conduce a mirar los objetivos.

Los educadores sabemos la importancia de que un alumno reconozca si asimiló bien o no los contenidos. Evaluarlos dependerá de un sinnúmero de actividades relacionales, que no se circunscriben a un examen, pregunta o problema a resolver. La interacción con el alumno, leer o escuchar sus preguntas, los aportes que genera en los espacios compartidos con sus pares, son algunos de los elementos que permiten al docente reconocer la apropiación de los contenidos o no. El examen escrito, el aporte cara a cara, el comentario en un foro, la evaluación en formato digital, etc., son solo medios para chequear el aprendizaje. 


\section{¿Una modalidad o un nuevo paradigma?}

A lo largo de este trabajo venimos señalando fortalezas y debilidades, y semejanzas y diferencias de la modalidad virtual o la tradicional modalidad presencial. Aclaramos que, desde hace un tiempo, el blend o la mixtura de ambas modalidades es un hecho y ha dado muy buenos resultados, siempre que haya claridad acerca del fin último.

Sin embargo, la pandemia y su obligación de aislamiento preventivo obligó a adoptar no solo una modalidad sino a abordar un nuevo modo de entender la educación.

Un paradigma es una cosmovisión, un modo de abordar lo real. Y, como toda cosmovisión está integrada por muchos elementos, algunos que podrían parecer heterogéneos pero, en su conjunto, dan respuesta a la realidad. La pandemia puso fin al "quiero que me atiendas"; terminó con el teaching para dar paso al learning. Ya la escuela no provee el conocimiento solamente, sino que ahora también produce aprendizaje.

Es cierto que para muchos de nosotros el paradigma del learning siempre existió en nuestros corazones, aunque fuera seminalmente. La mayoría de los docentes deseamos que nuestros alumnos aprendan y que dicho aprendizaje sea exitoso. Sin embargo, aunque esos anhelos fueran genuinos, no fue sino hasta hoy, a raíz de esta crisis mundial, que los elementos de este paradigma se hicieron patentes y conscientes. En las actuales circunstancias, no hay chances para no considerar estos elementos, porque la virtualidad tiene la particularidad de dejar en evidencia su falta.

Epicuro o Nietzsche, cuando afirmaban que el saber humano es un conocimiento de y para la vida humana, a su modo planteaban la necesidad de un aula inteligente, capaz de ayudar a cada individuo a centrarse y descubrir sus saberes y potencialidades. Así entendida, la acción educativa destaca tareas como el aprender a pensar críticamente; aprender a vivir creativamente, para eliminar la rutina y desafiar los modos convencionales de hacer y pensar las cosas; aprender a elegir libre y responsablemente, y, finalmente, aprender a actuar éticamente. Obviamente, no es una tarea simple para un docente, cual sea la modalidad elegida; pero en el siglo XXI el estudiante exige que abordemos este desafío. En el aula convencional puede pasar inadvertido si los docentes no tomamos la posta; en el aula virtual es imposible. 
La presencialidad tiene lugar en un espacio delimitado y en un tiempo concreto, al que tienen acceso los alumnos, los docentes y eventualmente- algún directivo o supervisor. Lo que sucede en el espacio áulico tradicional, respecta de la labor docente y el compromiso del alumno, no es tan evidente para quien no comparte dicho espacio. Si bien un padre o directivo podría detectar falencias o aciertos en dichas labores, la imposibilidad de ser testigo directo de la relación enseñar-aprender genera un manto de incertidumbre.

En cambio, la educación a distancia necesita de la virtualidad. Y la virtualidad excede la esfera privada. Si bien las instituciones educativas poseen sus propias plataformas, a las que tienen acceso solo sus participantes, lo cierto es que a esa aula inteligente puede entrar el estudiante, el directivo, el padre o cualquier otra persona que el estudiante permita. Lo que antes sucedía en un espacio físico al que no había fácil acceso ahora amplió su horizonte. La asincronía permite volver sobre una explicación, rehacer una actividad o desarrollarla según tiempos personales. La labor docente de corrección, feedback, creatividad, utilización de recursos, también se pone de manifiesto, aun para actores no primarios. Y así como un post en una red social es compartido primariamente con nuestros contactos, y basta que uno de ellos lo reproduzca en otro contexto para que se aleje de nuestra jurisdicción y alcance, lo mismo sucede con una clase remota. Esta modalidad, propia de nuestro mundo globalizado y tecnológico, atraviesa también a la educación, y mientras para muchos puede parecerse a un temible "gran hermano" que vigila su labor, para otros es la oportunidad de visibilizar su enorme tarea.

Por otro lado, existe una gran cantidad de agentes implicados en la educación remota, además de los padres, alumnos, docentes y directivos. Pensemos en las librerías, las bibliotecas distantes geográficamente, catálogos digitales y demás recursos que amplían la formación académica. Ni que hablar (daría para otro trabajo) la cuestión de los derechos de generación de contenido, los derechos de autor, la administración de datos, etc.

En épocas de aislamiento social por el COVID-19, toman la palabra y difunden su experiencia aquellos proyectos culturales que funcionan exclusivamente online y ya asoman como parte de un creciente paradigma digital destinado a acercar el arte a cualquier 
persona, donde sea que esté, una modalidad que en el contexto de estos días invita a repensar qué aporte tiene para hacer la virtualidad y cuáles serán las consecuencias de esta situación inédita en nuestra vida cotidiana.

Inevitable repensar el vínculo que mantenemos con la tecnología en este período atípico: ¿Era el coronavirus -y no la cuarta revolución digital - lo que iba a provocar el colapso de los paradigmas de esta era? Al margen de las consecuencias económicas que trae esta situación sin precedentes, los detractores de la tecnología no pueden negar la importancia de las artes para paliar los efectos negativos del confinamiento, especialmente en estos días en que pareciera potenciarse como nunca la creatividad humana a través de propuestas que florecen vía web: Instagram live por muestras de arte, talleres de escritura, recitales, lecturas. (Ezquiaga, 2020)

El texto de Ezquiaga sobre nuestra realidad actual es claro. Vivimos inmersos entre proyectos digitales. Cada día, no solo el arte sino nuestras actividades cotidianas se encuentran atravesadas por la digitalidad, y las acciones remotas son cada vez más frecuentes: comprar, leer, hacer un paseo virtual por un sitio geográficamente distante, visitar museos, asistir a una obra de teatro o concierto, y hasta las celebraciones religiosas. En tiempos de pandemia, estas prácticas ya casi son obligatorias y lo más probable que es que hayan venido a nuestras vidas para instalarse con más firmeza. ¿Por qué no la educación?

Nuestro paradigma global actual, podríamos decir, es digital y no se reduce solamente a la comunicación, sino que, desde hace un tiempo, cumple con funciones sociales, políticas y hasta estéticas. La escuela no está al margen de esto y su éxito o fracaso respecto de los fines de la educación también están sujetos a esta modalidad, nueva para muchos, pero vigente desde hace un tiempo. Con lo cual la modalidad virtual parecería que no es solo una herramienta más, sino un modo de concebir los procesos de enseñanza, un modo de entender la relación alumno-docente.

\section{Reflexión final}

Todo lo expuesto no intentó solucionar ningún problema ni dar respuesta a alguna hipótesis, sino más bien reflexionar sobre un tema ac- 
tual que nos tomó casi por sorpresa. Resultó de vital importancia aclarar algunas cuestiones respecto de la educación, para distinguir entre la "educación de emergencia", que recurre a un medio virtual para estar al alcance de los alumnos, y el auténtico proceso enseñanza-aprendizaje a distancia. La exigencia del paso a la educación virtual no admitió, en muchos casos, preparaciones y seguramente habrá mucho por mejorar y cambiar. Por lo tanto, confundir una clase dictada por "Zoom" o la utilización de un blog - a través del cual se envían tareas para que realice el alumno- con la educación remota sería un despropósito.

Lo que se puso de manifiesto ante la necesidad de cambiar la modalidad -y pese a las resistencias o dificultades de alumnos y maestros con el uso de las tecnologías - es que las formas tradicionales de enseñanza habían "ocultado" el olvido del fin de todo proceso educativo. La exigencia del cambio de modalidad solo es posible y menos engorrosa si se tiene en claro el fin de la educación y la esencia de la relación educativa.

La urgencia por el uso de la enseñanza remota también visibilizó la desigualdad de capacitación para el uso de las tecnologías, el desconocimiento por parte de muchos de los actores (alumnos-docentes), la dificultad para acceder a recursos tecnológicos (conectividad, software, hardware, etc.), fruto de las desigualdades socioeconómicas, entre otras cuestiones. Todo esto sumado a un contexto de emergencia sanitaria, ya que la virtualidad de las clases no fue una opción, una modalidad a ir aplicando, sino una urgencia para no perder la escolaridad. El estudiante y el docente que hoy día dicta o recibe clases de modo remoto virtualmente, es una persona que está en su casa aislado, constreñido a un espacio físico con el resto de su familia - muchos de los cuales son también docentes y alumnos-, intentando no mezclar el proceso educativo con las emociones y la vulnerabilidad que el contexto provee.

En la gran mayoría de los casos, el proceso educativo a través de la virtualidad, en estas circunstancias, resultó, al mismo tiempo, solución y prueba, pues el mismo acto educativo que se plantea servirá como test.

Estamos de acuerdo en que esta situación global sanitaria no es el mejor escenario para aprender-enseñar y mucho menos para instaurar cambios radicales. Pero la virtualidad forma parte ya de nuestra vida 
diaria y, aunque sea compleja su instrumentalización, por los factores ya mencionados, no podemos negar que se está transformando en un nuevo paradigma educativo. La situación de "emergencia" a causa del covid-19 aceleró la implementación de estos modelos (no sin consecuencias).

Uno de los desafíos que trae este paradigma - y que forma parte de las nuevas profesiones que aportó el mundo digital- es la producción de contenidos. Desde hace muchos años asistimos al crecimiento de canales de YouTube, Instagram, Vimeo, entre otros, en los que los particulares proveen a la sociedad producciones propias. Gran cantidad de ellas conforman un cúmulo de tutoriales educativos, que aportan a la sociedad en general un conocimiento gratuito y dinámico. Sabemos que no todo lo que está en la red es de calidad, pero también tenemos experiencia de excelencia. ¿Qué pasaría si todo lo que producimos los docentes día a día en un aula física, con cada clase o explicación, ahora pasase a formar parte del acervo cultural de toda la sociedad?

Más allá de la discusión —de suma importancia— respecto de los derechos de generación de dicho contenido, es importante destacar estas prácticas como una posibilidad de mostrar y de compartir el saber. Se trata también de la oportunidad de entrar en diálogo con otros docentes que pueden enriquecer el pensamiento y, asimismo, respecto de producciones de alumnos y docentes que pueden trascender los límites geográficos y temporales.

Quienes nos dedicamos a la tarea académica tenemos espacios para dar a conocer nuestras investigaciones, pero ¡qué gran oportunidad, para un docente no académico, tener la posibilidad de mostrar, compartir y de que otros puedan adoptar un recurso que ha preparado para sus alumnos!

Asimismo, la virtualidad permite hoy que los alumnos compartan en espacios de la escuela y las instituciones educativas sus logros, ideas, propuestas y trabajos, y que no solo tenga cuenta de ellos su círculo familiar reducido, sino que puedan ser compartidos con su familia amplia.

Existen muchos factores involucrados en la enseñanza exitosa y efectiva en línea: desde el diseño del curso, las actividades, el contenido, la presencia docente del maestro, la interacción entre estu- 
diantes y el maestro, la autonomía del estudiante e incluso la gestión del tiempo. (Meinecke, 2020, s/p).

La opinión de quien suscribe este artículo es que este nuevo paradigma educativo, que está instalándose, visibilizó las falencias que ya existían en el proceso de enseñanza tradicional, falencias que no dependen de las modalidades, de los espacios físicos (aunque estos pueden ayudar) sino de entender concretamente de qué se trata enseñaraprender. No es la intención entonces descalificar o echar por tierra las clases presenciales tradicionales, que tanto aportan y han aportado en la formación de cada uno de nosotros.

La escuela funcionó bien con la cultura de la tiza, aún sigue funcionando con esta noble tecnología que probablemente no desaparezca del aula. La práctica de la enseñanza tomará en serio las tecnologías digitales cuando docentes y estudiantes, desde los fundamentos del saber enseñar y aprender que conocemos, encontremos en su uso nuevo sentido, conocimiento, y lo más importante más seguridad y más confianza. (Artópulos \& Lion, 2016, p. 19).

Yo elijo enseñar virtualmente.

\section{Referencias bibliográficas}

Artópulos, A. y Lion, C. (2016). La escuela de las pantallas. Ebook. Fundación Telefónica- Ariel.

Es-Slami, A. et al. (2015). El nuevo paradigma de la educación digital, Ebook, Recuperado de https://aunclicdelastic.blogthinkbig.com/wp-content/uploads/El-nuevo-paradigma-de-laeducaci\%C3\%B3n-digital.pdf

Ezquiaga, M. (2020). ¿Cambio de paradigma?: el coronavirus potencia los proyectos culturales que nacieron digitales. Recuperado de https://www.infobae.com/cultura/2020/03/25/cambiode-paradigma-el-coronavirus-potencia-los-proyectos-culturales-que-nacieron-digitales/

Maritain, J. (1943). Los fines de la Educación. Recuperado de http:// www.jacquesmaritain.com/pdf/10_EDU/01_ED_FinEdu. pdf

Meineke, M. (2020). ¿Cómo hacer una clase online en vivo efectiva y sin complicaciones? Recuperado de https://observatorio.tec. 
$\mathrm{mx} /$ edu-bits-blog/clase-online-efectiva-y-sin-complicaciones

Pelliccia, A. (2020). Coronavirus: qué tipo de escuela está generando la pandemia. Recuperado de https://www.perfil.com/noticias/columnistas/que-tipo-de-escuela-esta-generando-lapandemia.phtml

Watson, L. (2007). Building the future of learning. European Journal of Education, 42(2), 255-263. DOI:10.1111/j.14653435.2007.00299.x

Rocca, A. (2020). Aprendizaje virtual en cuarentena: el nuevo territorio de encuentro profesional. Recuperado de https://www.lanacion.com.ar/economia/aprendizaje-virtual-en-cuarentenael-nuevo-territorio-de-encuentro-profesional-nid2350511

Vigilante, A. (2020). Cos'è una classe virtuale (e perché deve essere libera). Recuperado de https://www.glistatigenerali.com/scuola/cose-una-classe-virtuale-e-perche-devessere-libera/ 